\title{
Organoleptic Properties of Guava Fruit Leather with Effectiveness of Increase in Storage Period
}

\author{
Er. Shaik Jakeer Basha*, A. Yerri Swamy, Lingala Ramu and D. Sreenivas \\ *Department Food Science and Technology, Mahatma Phule Krishi Vidyapeeth, Rahuri, India \\ *Corresponding author
}

\section{A B S T R A C T}

\begin{tabular}{|l|}
\hline Key w or d s \\
$\begin{array}{l}\text { Guava, Lalith, } \\
\text { Sardar, Leather, } \\
\text { storage }\end{array}$ \\
\hline Article Info \\
\hline $\begin{array}{l}\text { Accepted: } \\
18 \text { April } 2018 \\
\text { Available Online: } \\
\text { 10 May } 2018\end{array}$ \\
\hline
\end{tabular}

An investigation was carried out to study the effect of storage period on organoleptic properties of guava leather prepared from its cultivars of Sardar (white flesh) and Lalith (pink flesh). Preliminary experiments were conducted to find out the optimum levels of sugar, citric acid and salt for preparation of quality leather. The leather prepared was packed in butter paper and stored at ambient $\left(25+2{ }^{\circ} \mathrm{C}\right)$ and refrigerated temperature $\left(5+2^{0} \mathrm{C}\right)$ for 90 days to study their storage feasibility. Out of the seven treatments, best two $\mathrm{T}_{1}, \mathrm{~T}_{2}$ are selected for further storage period of 90 days by sensory panel members on 9 point hedonic scale rating. The stored samples were drawn periodically at 30 days interval for analysis. The guava leather $\left(625.76 \mathrm{~g} / \mathrm{kg}\right.$ pulp) was obtained from treatment $\mathrm{V}_{1} \mathrm{~T}_{1}$ $(750 \mathrm{~g}$ sugar $+5 \mathrm{~g}$ salt $+2 \mathrm{~g}$ citric acid $)$, followed by the other treatments $(618.06 \mathrm{~g} / \mathrm{kg}$ pulp $)$ in $\mathrm{V}_{1} \mathrm{~T}_{2}$ (750 $\mathrm{g}$ sugar) for Sardar guava variety, $\left(624.00 \mathrm{~g} / \mathrm{kg}\right.$ pulp) in $\mathrm{V}_{2} \mathrm{~T}_{1}(750 \mathrm{~g}$ sugar + $5 \mathrm{~g}$ salt $+2 \mathrm{~g}$ citric acid) and $\left(617.00 \mathrm{~g} / \mathrm{kg}\right.$ pulp) in $\mathrm{V}_{2} \mathrm{~T}_{2}(750 \mathrm{~g}$ sugar) for Lalith guava variety. The treatments $V_{1} T_{1}, V_{2} T_{1}$, and $V_{1} T_{2}, V_{2} T_{2}$ are same but mainly differ in cultivars of guava white and pink flesh. Chemical composition indicated that the fresh guava leather contained on an average 16.80 per cent moisture, $76.20^{\circ}$ Brix TSS, 14.36 per cent reducing sugars, 68.70 per cent total sugars, 0.541 per cent titrable acidity, $127.10 \mathrm{mg} / 100 \mathrm{~g}$ ascorbic acid. The guava leather prepared by using sugar, salt, citric acid (Treatment $\mathrm{V}_{1} \mathrm{~T}_{1}$ and $\mathrm{V}_{2} \mathrm{~T}_{1}$ ) were superior over the other treatments in respect of organoleptic properties. The mean score of fresh guava leather for colour and appearance was 8.60, flavor 8.50, texture 8.60 , taste 8.30 and overall acceptability 8.50 on 9 point Hedonic scale. The storage studies indicate that there was a gradual decrease in organoleptic properties of leather with advancement of storage period. Sensory quality of guava leather decreased at faster rate during storage, which was more at ambient temperature than refrigerated storage. Total microbial count was low initially but increased slightly during storage. However guava leather was found to be acceptable in good condition even after 90 days of storage at ambient and refrigerated temperature.

\section{Introduction}

Guava (Psidium guajava L.) is a member of dicotyledonous, belong to large member of Myrtaceae or Myrtle family believed to be originated in Central America and Southern part of Mexico (Somogyi et al., 1996). It is a small tree or shrub of 2 to $8 \mathrm{~m}$ in height with 
wide spreading branches (Singh 1988). In India it is claimed to be the fourth most important cultivated fruit in both area and production wise after mango, banana and citrus (Singhal, 1996). Whole guava fruit is edible along with skin, quite hardy, prolific bearer with sweet aroma and pleasant sour sweet taste, considered as one of most delicious and luxurious fruits, often marketed as "Super fruits".

The quality and nutritional value of guava fruits are influenced by physical and biochemical changes during maturation by photosynthesis and accumulation. Fully mature guava fruits have very strong flavour therefore it is unsuitable to use as a table purpose. The fruit has about $83 \%$ moisture and is an excellent source of ascorbic acid (100$260 \mathrm{mg} / 100 \mathrm{~g}$ pulp) and pectin $(0.5-1.8 \%)$ (Verma and Shrivastava, 1965), but has low energy (66 Cal/100 g) and protein content (1\%) (Bose et al., 1999). The fruit is rich in minerals like phosphorous (23-37 mg/100 g), calcium (14-30 mg/100 g), iron (0.6-1.4 $\mathrm{mg} / 100 \mathrm{~g}$ ), as well as vitamins like Niacin, Pantothenic acid, Thiamine, Riboflavin, vitamin A (Bose et al., 1999).. Guava is normally consumed fresh as a desert fruit can be processed into juice, nectar, pulp, jam, jelly, slices in syrup, fruit bar or dehydrated products, as well as being used as an additive to other fruit juices or pulps (Leite et al., 2006). Excellent salad, pudding, jam, jelly, cheese, canned fruit, RTS, nectar, squash, ice cream and toffees are made from guava (Jain and Asati, 2004). However, guava is highly perishable and cannot be stored for longer period. Moreover considerable proportion of the produce is lost during post-harvest linkage (Ahire, 1989). It is, therefore, imperative to develop suitable technology for preservation and processing of such surplus produce. There has been greater increase in the production rate of these fruits over the years, and this may be due to their increased consumption pattern in the tropics (FAO, 1983). It is common experience that $20-25 \%$ of the fruit is completely damaged and spoiled before it reaches the consumer (Yadav, 1997). Therefore, to utilize the produce at the time of glut and to save it from spoilage; the development of low cost processing technology of guava is highly required. It will also generate enough opportunities of selfemployment by starting small scale processing unit or cottage industry that will be remunerative to the growers. Thus the preparations of guava pulp with simple technology and its utilization in the form of pulp and leather have a great scope.

Fruit leathers are dehydrated fruit based products. They are a tasty, chewy, dried fruit product. Fruit leathers are made by pouring pureed fruit onto a flat surface for drying. When dried the fruit is pulled from the surface and rolled, it gets the name "Leather" from the fact that when the pureed fruit is dried, it is shiny and has the texture of leather. Due to its novel and attractive structure, and for being products that do not require refrigeration, they constitute a practical way to incorporate fruit solids, especially for children and adolescents. Fruit leathers allow leftover ripe fruits to be preserved. Therefore guava leather from two kinds of fully ripened guava fruits one is of white flesh of Sardar variety and another of pink flesh Lalith fruits is prepared and kept for storage period of 3 months for organoleptic studies at both ambient and refrigerated storage.

\section{Materials and Methods}

\section{Raw materials}

Well-matured, healthy, uniform sized over ripen fruits of local Lalith of pink and Sardar or Lucknow-49 of white flesh cultivars were collected from the Department of Horticulture and progressive farmers of the Rahuri, Nasik, Yeola Tahashils. 


\section{Ingredients}

Citric acid, salt, sugar and hydrogenated fat were obtained from market and used as ingredients for preparation of guava leather (Table 1).

\section{Chemicals}

Most of the chemicals used in this investigation were of analytical grade, obtained from M/s. British Drug House Mumbai, M/s. Sarabhai M. Chemicals, M/s. Baroda, S.D. Fine Chemical Ltd., Mumbai and E. Merck (India), Mumbai.

\section{Preparation of guava leathers}

Fine guava fruit pulp was used for the preparation of fruit leather. In this pulp content ingredient like sugar, salt and citric acid as per the formula, which are further mixed well and then smeared with vegetable oil on the aluminum or stainless steel trays into thin layers $(0.5$ to $1.0 \mathrm{~cm}$ thick). Then the pulp was dried in hot air oven at $50{ }^{0} \mathrm{C}$ for 8 $10 \mathrm{hrs}$. After that semi surface-dried pulp sheets were cut into desired size usually of rectangular and again dried for 8-10 hrs. After drying three layers of sheets were kept together and pressed properly to form one sheet. Then desired size $(3 \times 4 \mathrm{~cm})$ cutting was done and dried under fan for 2-3 hrs and then wrapped into a metalized polyester wrapper and then kept in plastic bag for storage study.

\section{Flow sheet for preparation of guava leather}

Over ripen guava fruits

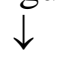

Thoroughly washing under running water

Extraction of fine pulp by pulp extractor

Addition of Ingredients

Smearing of trays with vegetable oil
Spreading of fine pulp in thin layer

(0.5-1.0 cm on smeared trays)

Initial drying for 8-10 hrs

Cutting surface dried pulp as per required size

$\downarrow$

Final drying for 8-10 hrs

Triple dried layers pressed together

$\downarrow$

Cutting dried sheets

$\downarrow$

Wrapping in butter paper

Filling in dry plastic jars

Sealing and storage

Standardization of ingredient levels for guava leather

Preliminary experiments were conducted to select the optimum level of each ingredient like sugar, salt, citric acid. The optimum levels of ingredients were finalized by sensory evaluation of guava leather by a panel of minimum ten semi-trained judges using 9 points Hedonic scale (Amerine et al., 1965).

\section{Packaging}

The prepared leathers were packed in a butter paper stored at both ambient $\left(25 \pm 2^{\circ} \mathrm{C}\right)$ and refrigerated $\left(7 \pm 2^{\circ} \mathrm{C}\right)$ temperature safely in laboratory at the middle compartment of the refrigerator for 3 months storage study. Analysis of stored guava leathers was carried out at an interval of $0,30,60,90$ day's storage period.

\section{Physico-chemical analysis}

The over ripen guava fruit pulp was analyzed for the moisture, TSS, titrable acidity, reducing sugars, total sugars, and vitamin $\mathrm{C}$ using standard methods of AOAC (2005). 


\section{Statistical analysis}

Results and experiments were planned and carried out using Factorial Completely Randomized Design (FCRD) using three to ten replications according to methods of the procedure given by Panse and Sukhatme (1967).

\section{Results and Discussion}

\section{Physio-chemical characteristics of Sardar guava fruit and pulp}

The physio-chemical composition of fruit plays a very important role in processing technology of guava as well as final quality of the product. The Physio-chemical composition of Sardar cultivar of guava is presented in Table 2. The over ripened fruits were round, yellowish in color. The average weight of fruit was $139 \mathrm{~g} /$ fruit. The average values for recovery of pulp and processing losses were 92.60 and 7.40 per cent, respectively.

\section{Physio-chemical characteristics of Lalith guava fruit and its pulp}

Lalith fruits were attractive, saffron yellow with occasional red blush and medium sized with firm pink colored flesh. It has good blend of sugar and acid and suitable for both processing and table purpose. Its yield was more than 24 per cent than the Allahabad Safeda variety (Yadav, 2007). The over ripen fruits of Lalith were round, yellowish in color. The average weight of fruit was $126 \mathrm{~g} /$ fruit. The average values for recovery of pulp and processing losses were 91.0 and 9.0 per cent, respectively.

\section{Recovery of pulp}

The recovery of pulp from Sardar guava variety was $92.60 \%$, while recovery of pulp for Lalith guava variety was $91.0 \%$ (Table 6).

\section{Organoleptic properties of pretreatment guava leathers}

From the organoleptic evaluation presented in Tables 3, 4 treatments $T_{2}$ and $T_{5}$ were selected as best among the 7 various treatments for both Sardar and Lalith guava leathers. Selected treatments $T_{2}$ and $T_{5}$ were renamed as $V_{1} T_{1}$ and $V_{1} T_{2}$ in Sardar guava leather, and for Lalith guava leather as $V_{2} T_{1}$ and $V_{2} T_{2}$.

\section{Organoleptic properties of fresh guava leathers (selected treatments)}

The selected treatments leather was prepared for further study. The organoleptic evaluations of fresh guava leather from both varieties are presented in Table 5. During organoleptic evaluation, $V_{1} T_{1}$ and $V_{2} T_{1}$ found similar and better than $V_{1} T_{2}$ and $V_{2} T_{2}$ for overall acceptability. So again $T_{2}$ and $T_{5}$ samples of guava leather (both varieties) are prepared and kept for storage of 3 months at both ambient and refrigerated conditions.

Yield and chemical properties of fresh guava leathers

The yield and chemical properties of fresh guava leather of both Sardar as well as Lalith cultivars are shown in Table 6. The yield of guava leathers ranged from $617-625 \mathrm{~g} / \mathrm{kg}$ of pulp. The yield of guava leather $\mathrm{V}_{1} \mathrm{~T}_{1}$ was slightly higher as compared to $V_{1} T_{2}, V_{2} T_{1}$, and $\mathrm{V}_{2} \mathrm{~T}_{2}$. There was no much difference in yield between three treatments, as the levels of ingredients are same.

\section{Changes in chemical composition of guava leathers during storage}

Guava leather prepared from selected treatments from both varieties was kept for storage study at ambient $\left(27 \pm 2^{\circ} \mathrm{C}\right)$ and refrigerator $\left(7 \pm 2^{\circ} \mathrm{C}\right)$ temperatures. The storage study results of guava leather are presented in Tables 9 to 12 . 


\section{Chemical properties of guava leathers}

Chemical properties of guava leathers are mentioned in Table 6. There was slight variation in chemical properties which might be due to change in variety. Pink flesh guava leather has low amount of ascorbic content when compared to the sardar guava leather.

Changes on organoleptic properties of guava leathers during storage

The freshly prepared guava leathers were evaluated organoleptically by a panel of semi trained judges on a 9 point Hedonic scale. The mean sensory scores of fresh and stored guava leather samples for parameters like color and appearance, texture, flavor, taste and overall acceptability of guava leather samples are presented in Tables 9-12.

\section{Color and appearance}

The score for color and appearance in the present investigation of guava leather as influenced by storage temperature and period is presented in Tables 9-12. A gradual decrease in score from 8.35 to 7.45 at ambient temperature and from 8.35 to 7.80 at refrigerated temperature was observed for 90 days of storage. The score 8.35 was observed in $\mathrm{V}_{1} \mathrm{~T}_{1}$ guava leather sample stored at refrigerated condition. Similar trend for color and appearance of guava leathers was observed at ambient condition but the values were at lower level than the refrigerated storage. The color deterioration was more in guava leathers stored at ambient condition. This may be due to degradation of pigments that might have occurred at ambient temperature.

Similar observations were reported in sweet potato leather (Collins and Hutsell, 1987), jack fruit leather (Che Man and Taufik, 1995), fig and other fruit products (Doreyappa Gowda $e t$ al., 1995), mango fruit bar with respect to storage temperature (Doreyappa Gowda et al., 1995), dried figs (Palve, 2002), guava-papaya fruit bar (Vennilla et al., 2004), fig leather (Chandeswar et al., 2004 and Kotlawar, 2008) and high protein tamarind leather (Kharche, 2012). The results obtained in the present investigation are in concurrent with the literature.

\section{Flavor}

The results on flavor score of guava leather samples as influenced by storage are presented in Tables 9-12. A gradual decrease in score for flavor from 8.20 to 7.55 at ambient temperature and from 8.20 to 7.62 at refrigerated temperature was observed. The flavor retention was higher at refrigerated condition than the ambient condition. In both storage conditions treatment $\mathrm{V}_{2} \mathrm{~T}_{1}$ produced highest flavor score when stored at refrigerated condition. The flavoring compounds may be lost at higher rate at higher storage temperature (thus causing lower flavor score) at ambient condition than at refrigerated condition.

A gradual decrease in flavor score was reported with increase in storage period of guava leathers. Dried figs (Mali, 1997 and Chandeswar et al., 2004), guava-Papaya fruit bar (Vennilla et al., 2004), fig leather (Kotlawar, 2008) and high protein tamarind leather (Kharche, 2012). The results obtained in the present investigation are similar to earlier reports.

\section{Texture}

The sensory results for texture score of guava leather samples are presented in Tables 9-12. A gradual decrease in texture score was observed in guava leathers from 8.27 to 7.56 at ambient temperature and from 8.27 to 7.80 at refrigerated temperature. 
Table.1 Treatment details for experimentation

\begin{tabular}{|c|c|c|c|c|}
\hline Treatments & Pulp (\%) & Sugar $(\boldsymbol{\%})$ & Salt $(\%)$ & Citric acid (\%) \\
\hline $\mathbf{T}_{\mathbf{1}}$ & 100 & 500 & 5 & 2 \\
\hline $\mathbf{T}_{\mathbf{2}}$ & 100 & 750 & 5 & 2 \\
\hline $\mathbf{T}_{\mathbf{3}}$ & 100 & 1000 & 5 & 2 \\
\hline $\mathbf{T}_{\mathbf{4}}$ & 100 & 750 & 5 & 4 \\
\hline $\mathbf{T}_{\mathbf{5}}$ & 100 & 750 & - & - \\
\hline $\mathbf{T}_{\mathbf{6}}$ & 100 & 750 & 5 & - \\
\hline $\mathbf{T}_{\mathbf{7}}$ & 100 & 750 & - & 4 \\
\hline
\end{tabular}

Table.2 Physio-chemical characteristics of Sardar, Lalith guava fruits

\begin{tabular}{|c|c|c|c|}
\hline S. No. & Parameters & Sardar (white flesh) & Lalith (Pink flesh) \\
\hline A. & \multicolumn{3}{|c|}{ Physical parameters of fruits } \\
\hline 1. & Shape & Round & Round \\
\hline 2. & Color & Yellow & Saffron yellow \\
\hline 3. & Average length $(\mathrm{cm})$ & 6.20 & 4.10 \\
\hline 4. & Average fruit weight $(\mathrm{g})$ & 139.0 & 126 \\
\hline 5. & Diameter $(\mathrm{cm})$ & 6.20 & 6.2 \\
\hline 6. & Per cent of pulp recovery $(\%)$ & 92.60 & 91.0 \\
\hline 7. & $\begin{array}{l}\text { Waste material/Seed content } \\
\text { losses }(\%)\end{array}$ & 7.40 & 9.0 \\
\hline B. & \multicolumn{3}{|c|}{ Chemical constituents of Pulp } \\
\hline 1. & TSS $\left({ }^{\circ}\right.$ Brix $)$ & 9.20 & 9.10 \\
\hline 2. & Acidity $(\%)$ & 0.450 & 0.380 \\
\hline 3. & Total sugars (\%) & 7.70 & 5.10 \\
\hline 4. & Reducing sugars (\%) & 5.30 & 7.40 \\
\hline 5. & Vitamin C (mg/100 g) & 210 & 130 \\
\hline 6. & Moisture (\%) & 82.56 & 83.60 \\
\hline
\end{tabular}

Table.3 Organoleptic evaluation of fresh Sardar variety guava leather ${ }^{\mathrm{a}}$

\begin{tabular}{|l|c|c|c|c|c|c|l|}
\hline $\begin{array}{l}\text { S. } \\
\text { No }\end{array}$ & Treatments & $\begin{array}{l}\text { Color and } \\
\text { appearance }\end{array}$ & Flavor & Taste & Texture & $\begin{array}{l}\text { Overall } \\
\text { acceptability }\end{array}$ & $\begin{array}{l}\text { Selected for } \\
\text { further study }\end{array}$ \\
\hline $\mathbf{1}$ & $\mathrm{V}_{1} \mathrm{~T}_{1}$ & 7.60 & 8.00 & 8.00 & 7.90 & 7.87 & Not selected \\
\hline $\mathbf{2}$ & $\mathrm{V}_{1} \mathrm{~T}_{2}$ & 8.80 & 8.30 & 8.20 & 8.30 & $\mathbf{8 . 4 0}$ & $\mathbf{V}_{\mathbf{1}} \mathbf{T}_{\mathbf{1}}$ selected \\
\hline $\mathbf{3}$ & $\mathrm{V}_{1} \mathrm{~T}_{3}$ & 8.00 & 7.70 & 7.60 & 8.10 & 7.85 & Not selected \\
\hline $\mathbf{4}$ & $\mathrm{V}_{1} \mathrm{~T}_{4}$ & 7.90 & 7.50 & 7.60 & 7.60 & 7.65 & Not selected \\
\hline $\mathbf{5}$ & $\mathrm{V}_{1} \mathrm{~T}_{5}$ & 8.40 & 7.80 & 8.00 & 7.80 & $\mathbf{8 . 0 0}$ & $\mathbf{V}_{\mathbf{1}} \mathbf{T}_{\mathbf{2}}$ selected \\
\hline $\mathbf{6}$ & $\mathrm{V}_{1} \mathrm{~T}_{6}$ & 6.50 & 6.40 & 6.50 & 6.90 & 6.57 & Not selected \\
\hline $\mathbf{7}$ & $\mathrm{V}_{1} \mathrm{~T}_{7}$ & 6.70 & 6.90 & 6.90 & 6.80 & 6.82 & Not selected \\
\hline
\end{tabular}

Whereas, $a=$ Ten replications with 9 point hedonic scale

$\mathrm{V}_{1}=$ Sardar guava variety (white flesh) 
Table.4 Organoleptic evaluation of fresh Lalith variety guava leather ${ }^{\mathrm{a}}$

\begin{tabular}{|l|c|c|c|c|c|l|l|}
\hline $\begin{array}{l}\text { S. } \\
\text { No }\end{array}$ & Treatments & $\begin{array}{l}\text { Color and } \\
\text { appearance }\end{array}$ & Flavor & Taste & Texture & $\begin{array}{l}\text { Overall } \\
\text { acceptability }\end{array}$ & $\begin{array}{l}\text { Selected for } \\
\text { further study }\end{array}$ \\
\hline $\mathbf{1}$ & $\mathrm{V}_{2} \mathrm{~T}_{1}$ & 7.5 & 8.0 & 7.5 & 7.6 & 7.65 & Not selected \\
\hline $\mathbf{2}$ & $\mathrm{V}_{2} \mathrm{~T}_{2}$ & 8.7 & 8.2 & 8.2 & 8.7 & $\mathbf{8 . 4 5}$ & $\mathbf{V}_{2} \mathbf{T}_{1}$ selected \\
\hline $\mathbf{3}$ & $\mathrm{V}_{2} \mathrm{~T}_{3}$ & 8.5 & 8.2 & 8.5 & 8.0 & 8.30 & Not selected \\
\hline $\mathbf{4}$ & $\mathrm{V}_{2} \mathrm{~T}_{4}$ & 7.8 & 8.0 & 8.0 & 7.9 & 7.92 & Not selected \\
\hline $\mathbf{5}$ & $\mathrm{V}_{2} \mathrm{~T}_{5}$ & 8.5 & 8.2 & 8.2 & 8.4 & $\mathbf{8 . 3 3}$ & $\mathbf{V}_{2} \mathbf{T}_{2}$ selected \\
\hline $\mathbf{6}$ & $\mathrm{V}_{2} \mathrm{~T}_{6}$ & 7.6 & 7.9 & 8.3 & 7.6 & 7.85 & Not selected \\
\hline $\mathbf{7}$ & $\mathrm{V}_{2} \mathrm{~T}_{7}$ & 7.7 & 8.0 & 8.0 & 7.8 & 7.87 & Not selected \\
\hline
\end{tabular}

Whereas,

$\mathrm{a}=$ Ten replications with 9 point hedonic scale

$\mathrm{V}_{2}=$ Lalith guava variety (pink flesh)

Table.5 Organoleptic properties of selected fresh guava leathers ${ }^{\mathrm{a}}$

\begin{tabular}{|c|c|c|c|c|c|}
\hline Treatments & $\begin{array}{c}\text { Color and } \\
\text { appearance }\end{array}$ & Flavor & Texture & Taste & $\begin{array}{c}\text { Overall } \\
\text { acceptability }\end{array}$ \\
\hline $\mathbf{V}_{\mathbf{1}} \mathbf{T}_{\mathbf{1}}$ & 8.59 & 8.46 & 8.39 & 8.64 & 8.53 \\
\hline $\mathbf{V}_{\mathbf{1}} \mathbf{T}_{\mathbf{2}}$ & 8.14 & 8.03 & 8.14 & 8.12 & 8.07 \\
\hline $\mathbf{V}_{\mathbf{2}} \mathbf{T}_{\mathbf{1}}$ & 8.65 & 8.52 & 8.66 & 8.32 & 8.56 \\
\hline $\mathbf{V}_{\mathbf{2}} \mathbf{T}_{\mathbf{2}}$ & 8.05 & 7.80 & 7.90 & 8.13 & 8.37 \\
\hline $\mathbf{S E m} \pm$ & 0.022 & 0.019 & 0.022 & 0.016 & 0.018 \\
\hline $\mathbf{C D}$ at $\mathbf{5} \%$ & 0.068 & 0.059 & 0.068 & 0.049 & 0.055 \\
\hline
\end{tabular}

Whereas, $\mathrm{a}=$ Four replications.

$\mathrm{V}_{1:}$ Sardar guava variety (white flesh), $\mathrm{V}_{2}$ : Lalith guava variety (Pink flesh)

$\mathrm{T}_{1:} 750 \mathrm{~g}$ sugar $+5 \mathrm{~g}$ salt $+2 \mathrm{~g}$ citric acid per kg guava pulp

$\mathrm{T}_{2:} 750 \mathrm{~g}$ sugar per kg guava pulp

Table.6 Yield and chemical properties of fresh guava leathers ${ }^{\mathrm{a}}$

\begin{tabular}{|c|c|c|c|c|c|c|c|c|}
\hline $\begin{array}{l}\text { Treat } \\
\text { ments }\end{array}$ & $\begin{array}{l}\text { Yield } \\
\text { (g/kg } \\
\text { Pulp) }\end{array}$ & $\begin{array}{c}\text { Moisture } \\
(\%)\end{array}$ & $\begin{array}{c}\text { TSS } \\
\left({ }^{\mathbf{0}} \text { Brix }\right)\end{array}$ & $\begin{array}{c}\text { Titrable } \\
\text { acidity } \\
(\%)\end{array}$ & $\begin{array}{c}\text { Reducing } \\
\text { sugars } \\
\text { (\%) }\end{array}$ & $\begin{array}{c}\text { Total } \\
\text { sugars } \\
(\%)\end{array}$ & $\begin{array}{l}\text { Ascorbic } \\
\text { acid } \\
(\mathrm{mg} / 100 \mathrm{l})\end{array}$ & $\begin{array}{l}\text { Total } \\
\text { cost }\end{array}$ \\
\hline$V_{1} T_{1}$ & 625.76 & 15.29 & 76.10 & 0.541 & 14.32 & 68.72 & 125.28 & 135.55 \\
\hline$V_{1} T_{2}$ & 618.06 & 15.12 & 76.00 & 0.462 & 14.12 & 68.23 & 127.30 & 135.00 \\
\hline $\mathbf{V}_{2} \mathbf{T}_{1}$ & 624.00 & 16.75 & 75.85 & 0.490 & 14.19 & 68.47 & 71.81 & 155.55 \\
\hline $\mathbf{V}_{2} \mathbf{T}_{2}$ & 617.00 & 16.27 & 75.85 & 0.412 & 12.92 & 68.28 & 73.34 & 155.00 \\
\hline SEm \pm & 1.711 & 0.024 & 0.036 & 0.0011 & 0.014 & 0.010 & 0.127 & - \\
\hline CD at $5 \%$ & NS & 0.073 & NS & NS & 0.045 & 0.032 & NS & - \\
\hline
\end{tabular}

Whereas, $\mathrm{a}=$ Four replications.

$\mathrm{V}_{1:}$ Sardar guava variety (white flesh), $\mathrm{V}_{2:}$ Lalith guava variety (Pink flesh)

$\mathrm{T}_{1:} 750 \mathrm{~g}$ sugar $+5 \mathrm{~g}$ salt $+2 \mathrm{~g}$ citric acid per kg guava pulp

$\mathrm{T}_{2:} 750 \mathrm{~g}$ sugar per kg guava pulp. 
Table.8 Texture analysis of guava leathers

\begin{tabular}{|c|c|c|c|c|c|}
\hline Treatments & $\begin{array}{l}\text { Colour and } \\
\text { appearance }\end{array}$ & Flavour & Texture & Taste & $\begin{array}{c}\text { Overall } \\
\text { acceptability }\end{array}$ \\
\hline \multicolumn{6}{|l|}{ Variety } \\
\hline $\mathbf{V}_{1}$ & 8.36 & 8.24 & 8.27 & 8.38 & 8.30 \\
\hline $\mathbf{V}_{2}$ & 8.35 & 8.16 & 8.28 & 8.22 & 8.46 \\
\hline $\operatorname{SEm}( \pm)$ & 0.015 & 0.013 & 0.015 & 0.011 & 0.012 \\
\hline CD@ 5\% & NS & 0.041 & NS & 0.035 & 0.039 \\
\hline \multicolumn{6}{|l|}{ Treatments } \\
\hline $\mathrm{T}_{1}$ & 8.62 & 8.49 & 8.52 & 8.48 & 8.54 \\
\hline $\mathbf{T}_{2}$ & 8.09 & 7.92 & 8.02 & 8.13 & 8.22 \\
\hline $\operatorname{SEm}( \pm)$ & 0.015 & 0.013 & 0.015 & 0.011 & 0.012 \\
\hline CD@ $9 \%$ & 0.048 & 0.041 & 0.048 & 0.035 & 0.039 \\
\hline \multicolumn{6}{|c|}{ Two factor interaction } \\
\hline $\mathbf{V}_{1} \mathbf{T}_{1}$ & 8.59 & 8.46 & 8.39 & 8.64 & 8.53 \\
\hline $\mathbf{V}_{1} \mathbf{T}_{2}$ & 8.14 & 8.03 & 8.14 & 8.12 & 8.07 \\
\hline $\mathbf{V}_{2} \mathbf{T}_{1}$ & 8.65 & 8.52 & 8.66 & 8.32 & 8.56 \\
\hline $\mathbf{V}_{2} \mathbf{T}_{2}$ & 8.05 & 7.80 & 7.90 & 8.13 & 8.37 \\
\hline $\operatorname{SEm}( \pm)$ & 0.022 & 0.019 & 0.022 & 0.016 & 0.018 \\
\hline CD@ @ $\%$ & 0.068 & 0.050 & 0.068 & 0.049 & 0.055 \\
\hline
\end{tabular}

Whereas,

$\mathrm{AT}=$ Ambient temperature, $\mathrm{RT}=$ Refrigerated temperature

$\mathrm{V}_{1:}$ Sardar guava variety (white flesh), $\mathrm{V}_{2}$ : Lalith guava variety (Pink flesh)

$\mathrm{T}_{1}: 750 \mathrm{~g}$ sugar $+5 \mathrm{~g}$ salt $+2 \mathrm{~g}$ citric acid per kg guava pulp

$\mathrm{T}_{2}: 750 \mathrm{~g}$ sugar per kg guava pulp. 
Table.9 Effect of storage period on organoleptic properties of fresh guava leathers at 0 days storage

\begin{tabular}{|c|c|c|c|c|c|}
\hline Treatments & $\begin{array}{l}\text { Colour and } \\
\text { appearance }\end{array}$ & Flavour & Texture & Taste & $\begin{array}{c}\text { Overall } \\
\text { acceptability }\end{array}$ \\
\hline \multicolumn{6}{|l|}{ Variety } \\
\hline $\mathbf{V}_{1}$ & 8.36 & 8.24 & 8.27 & 8.38 & 8.30 \\
\hline $\mathbf{V}_{2}$ & 8.35 & 8.16 & 8.28 & 8.22 & 8.46 \\
\hline $\operatorname{SEm}( \pm)$ & 0.015 & 0.013 & 0.015 & 0.011 & 0.012 \\
\hline CD@ $@$ \%\% & NS & 0.041 & NS & 0.035 & 0.039 \\
\hline \multicolumn{6}{|l|}{ Treatments } \\
\hline $\mathrm{T}_{1}$ & 8.62 & 8.49 & 8.52 & 8.48 & 8.54 \\
\hline $\mathbf{T}_{2}$ & 8.09 & 7.92 & 8.02 & 8.13 & 8.22 \\
\hline $\operatorname{SEm}( \pm)$ & 0.015 & 0.013 & 0.015 & 0.011 & 0.012 \\
\hline CD@ $@ 5 \%$ & 0.048 & 0.041 & 0.048 & 0.035 & 0.039 \\
\hline \multicolumn{6}{|c|}{ Two factor interaction } \\
\hline $\mathbf{V}_{1} \mathbf{T}_{1}$ & 8.59 & 8.46 & 8.39 & 8.64 & 8.53 \\
\hline $\mathbf{V}_{1} \mathbf{T}_{2}$ & 8.14 & 8.03 & 8.14 & 8.12 & 8.07 \\
\hline $\mathbf{V}_{2} \mathbf{T}_{1}$ & 8.65 & 8.52 & 8.66 & 8.32 & 8.56 \\
\hline $\mathbf{V}_{2} \mathbf{T}_{2}$ & 8.05 & 7.80 & 7.90 & 8.13 & 8.37 \\
\hline $\operatorname{SEm}( \pm)$ & 0.022 & 0.019 & 0.022 & 0.016 & 0.018 \\
\hline CD@ @ & 0.068 & 0.050 & 0.068 & 0.049 & 0.055 \\
\hline
\end{tabular}

$\mathrm{A}=$ Ambient $\left(25 \pm 2{ }^{\circ} \mathrm{C}\right), \mathrm{R}=$ Refrigerated $\left(5 \pm 2{ }^{\circ} \mathrm{C}\right)$

$\mathrm{V}_{1:}$ Sardar guava variety (white flesh), $\mathrm{V}_{2}$ : Lalith guava variety (Pink flesh).

$\mathrm{T}_{1}: 750 \mathrm{~g}$ sugar $+5 \mathrm{~g}$ salt $+2 \mathrm{~g}$ citric acid per kg guava pulp, $\mathrm{T}_{2}: 750 \mathrm{~g}$ sugar per kg guava pulp. 
Table.10 Effect of storage period on organoleptic properties of guava leather at 30 days storage

\begin{tabular}{|c|c|c|c|c|c|c|c|c|c|c|}
\hline \multirow[t]{2}{*}{ Treatments } & \multicolumn{2}{|c|}{$\begin{array}{l}\text { Colour and } \\
\text { appearance }\end{array}$} & \multicolumn{2}{|c|}{ Flavour } & \multicolumn{2}{|c|}{ Texture } & \multicolumn{2}{|c|}{ Taste } & \multicolumn{2}{|c|}{$\begin{array}{c}\text { Overall } \\
\text { acceptability }\end{array}$} \\
\hline & A & $\mathrm{R}$ & A & $\mathrm{R}$ & A & $\mathrm{R}$ & A & $\mathrm{R}$ & A & $\mathrm{R}$ \\
\hline \multicolumn{11}{|l|}{ Variety } \\
\hline $\mathrm{V}_{1}$ & 7.89 & 8.20 & 7.75 & 7.90 & 7.93 & 8.02 & 8.00 & 8.22 & 7.89 & 8.03 \\
\hline $\mathbf{V}_{2}$ & 7.93 & 7.93 & 7.79 & 7.97 & 7.86 & 8.08 & 8.10 & 8.18 & 8.11 & 8.23 \\
\hline $\operatorname{SEm}( \pm)$ & 0.012 & 0.015 & 0.019 & 0.018 & 0.015 & 0.010 & 0.019 & 0.020 & 0.016 & 0.016 \\
\hline CD@5\% & 0.038 & 0.048 & NS & 0.058 & 0.047 & 0.030 & 0.061 & NS & 0.049 & 0.050 \\
\hline \multicolumn{11}{|l|}{ Treatments } \\
\hline $\mathrm{T}_{1}$ & 8.18 & 8.41 & 8.05 & 8.19 & 8.03 & 8.23 & 8.28 & 8.43 & 8.14 & 8.33 \\
\hline $\mathbf{T}_{2}$ & 7.64 & 7.72 & 7.48 & 7.67 & 7.76 & 7.87 & 7.81 & 7.97 & 7.86 & 7.93 \\
\hline $\operatorname{SEm}( \pm)$ & 0.012 & 0.015 & 0.019 & 0.018 & 0.015 & 0.010 & 0.019 & 0.020 & 0.016 & 0.016 \\
\hline CD@ 5\% & 0.038 & 0.048 & 0.060 & 0.058 & 0.047 & 0.030 & 0.061 & 0.063 & 0.049 & 0.050 \\
\hline \multicolumn{11}{|c|}{ Two factor interaction } \\
\hline $\mathbf{V}_{1} T_{1}$ & 8.12 & 8.56 & 7.97 & 8.08 & 8.04 & 8.12 & 8.45 & 8.61 & 8.13 & 8.33 \\
\hline $\mathbf{V}_{1} \mathbf{T}_{2}$ & 7.66 & 7.83 & 7.53 & 7.71 & 7.82 & 7.92 & 7.54 & 7.82 & 7.66 & 7.73 \\
\hline $\mathbf{V}_{2} \mathbf{T}_{1}$ & 8.24 & 8.25 & 8.14 & 8.30 & 8.02 & 8.34 & 8.12 & 8.25 & 8.16 & 8.34 \\
\hline $\mathbf{V}_{2} \mathbf{T}_{2}$ & 7.62 & 7.62 & 7.44 & 7.63 & 7.70 & 7.82 & 8.08 & 8.12 & 8.06 & 8.12 \\
\hline $\operatorname{SEm}( \pm)$ & 0.017 & 0.023 & 0.027 & 0.026 & 0.021 & 0.014 & 0.028 & 0.029 & 0.022 & 0.023 \\
\hline CD@ 5\% & 0.054 & 0.066 & 0.085 & 0.082 & 0.066 & 0.043 & 0.086 & 0.089 & 0.070 & 0.071 \\
\hline
\end{tabular}


Table.11 Effect of storage period on organoleptic properties of guava leather at 60 days storage

\begin{tabular}{|c|c|c|c|c|c|c|c|c|c|c|}
\hline \multirow[t]{2}{*}{ Treatments } & \multicolumn{2}{|c|}{$\begin{array}{l}\text { Colour and } \\
\text { appearance }\end{array}$} & \multicolumn{2}{|c|}{ Flavour } & \multicolumn{2}{|c|}{ Texture } & \multicolumn{2}{|c|}{ Taste } & \multicolumn{2}{|c|}{ Overall acceptability } \\
\hline & A & $\mathrm{R}$ & A & $\mathrm{R}$ & A & $\mathrm{R}$ & A & $\mathrm{R}$ & A & $\mathrm{R}$ \\
\hline \multicolumn{11}{|l|}{ Variety } \\
\hline $\mathrm{V}_{1}$ & 7.66 & 8.06 & 7.22 & 7.43 & 7.72 & 7.90 & 7.68 & 8.13 & 7.59 & 7.97 \\
\hline $\mathbf{V}_{2}$ & 7.78 & 7.76 & 7.59 & 7.73 & 7.59 & 7.83 & 7.95 & 8.18 & 7.82 & 8.06 \\
\hline $\operatorname{SEm}( \pm)$ & 0.012 & 0.014 & 0.021 & 0.011 & 0.008 & 0.011 & 0.014 & 0.011 & 0.013 & 0.009 \\
\hline CD@ 5\% & 0.038 & 0.045 & 0.066 & 0.035 & 0.025 & 0.035 & 0.044 & 0.036 & 0.041 & 0.029 \\
\hline \multicolumn{11}{|l|}{ Treatments } \\
\hline $\mathbf{T}_{1}$ & 8.06 & 8.22 & 7.63 & 7.78 & 7.80 & 8.00 & 8.05 & 8.43 & 7.87 & 8.25 \\
\hline $\mathbf{T}_{2}$ & 7.38 & 7.60 & 7.19 & 7.39 & 7.51 & 7.73 & 7.58 & 7.88 & 7.55 & 7.79 \\
\hline $\operatorname{SEm}( \pm)$ & 0.012 & 0.014 & 0.021 & 0.011 & 0.008 & 0.011 & 0.014 & 0.011 & 0.013 & 0.009 \\
\hline CD@ 5\% & 0.038 & 0.045 & 0.066 & 0.035 & 0.025 & 0.035 & 0.044 & 0.036 & 0.041 & 0.029 \\
\hline \multicolumn{11}{|c|}{ Two factor interaction } \\
\hline $\mathbf{V}_{1} \mathbf{T}_{1}$ & 8.00 & 8.33 & 7.39 & 7.62 & 7.82 & 7.98 & 8.02 & 8.63 & 7.84 & 8.40 \\
\hline $\mathbf{V}_{1} \mathbf{T}_{2}$ & 7.32 & 7.78 & 7.05 & 7.24 & 7.63 & 7.83 & 7.35 & 7.64 & 7.35 & 7.55 \\
\hline $\mathbf{V}_{2} \mathbf{T}_{1}$ & 8.12 & 8.11 & 7.86 & 7.94 & 7.79 & 8.03 & 8.08 & 8.24 & 7.90 & 8.10 \\
\hline $\mathbf{V}_{2} \mathbf{T}_{2}$ & 7.44 & 7.42 & 7.32 & 7.53 & 7.39 & 7.64 & 7.81 & 8.12 & 7.75 & 8.02 \\
\hline $\operatorname{SEm}( \pm)$ & 0.017 & 0.021 & 0.030 & 0.016 & 0.011 & 0.016 & 0.020 & 0.016 & 0.019 & 0.013 \\
\hline CD @ 5\% & NS & 0.064 & 0.094 & NS & 0.035 & 0.049 & 0.063 & 0.051 & 0.059 & 0.041 \\
\hline
\end{tabular}

A=Ambient $\left(25 \pm 2{ }^{0} \mathrm{C}\right), \mathrm{R}=$ Refrigerated $\left(5 \pm 2{ }^{0} \mathrm{C}\right)$

$\mathrm{V}_{1}$ : Sardar guava variety (white flesh), $\mathrm{V}_{2}$ : Lalith guava variety (Pink flesh).

$\mathrm{T}_{1}: 750 \mathrm{~g}$ sugar $+5 \mathrm{~g}$ salt $+2 \mathrm{~g}$ citric acid per kg guava pulp, $\mathrm{T}_{2}: 750 \mathrm{~g}$ sugar per $\mathrm{kg}$ guava pulp 
Table.12 Effect of storage period on organoleptic properties of guava leather at 90 days storage

\begin{tabular}{|c|c|c|c|c|c|c|c|c|c|c|}
\hline \multirow[t]{2}{*}{ Treatments } & \multicolumn{2}{|c|}{$\begin{array}{l}\text { Colour and } \\
\text { appearance }\end{array}$} & \multicolumn{2}{|c|}{ Flavour } & \multicolumn{2}{|c|}{ Texture } & \multicolumn{2}{|c|}{ Taste } & \multicolumn{2}{|c|}{$\begin{array}{c}\text { Overall } \\
\text { acceptability }\end{array}$} \\
\hline & A & $\mathrm{R}$ & A & $\mathrm{R}$ & A & $\mathrm{R}$ & A & $\mathrm{R}$ & A & $\mathrm{R}$ \\
\hline \multicolumn{11}{|l|}{ Variety } \\
\hline$V_{1}$ & 7.33 & 7.72 & 7.24 & 7.43 & 7.40 & 7.64 & 7.52 & 8.13 & 7.40 & 7.73 \\
\hline $\mathbf{V}_{2}$ & 7.58 & 7.87 & 7.87 & 7.82 & 7.72 & 7.97 & 7.47 & 7.83 & 7.67 & 7.83 \\
\hline $\operatorname{SEm}( \pm)$ & 0.012 & 0.009 & 0.013 & 0.011 & 0.012 & 0.019 & 0.009 & 0.012 & 0.014 & 0.014 \\
\hline CD@ $@ 5 \%$ & 0.039 & 0.030 & 0.041 & 0.035 & 0.038 & 0.059 & 0.027 & 0.038 & 0.043 & 0.044 \\
\hline \multicolumn{11}{|l|}{ Treatments } \\
\hline $\mathrm{T}_{1}$ & 7.68 & 8.02 & 7.73 & 7.78 & 7.65 & 7.99 & 7.71 & 8.28 & 7.72 & 8.00 \\
\hline $\mathbf{T}_{2}$ & 7.23 & 7.57 & 7.39 & 7.47 & 7.47 & 7.62 & 7.28 & 7.68 & 7.35 & 7.56 \\
\hline $\operatorname{SEm}( \pm)$ & 0.012 & 0.009 & 0.013 & 0.011 & 0.012 & 0.019 & 0.009 & 0.012 & 0.014 & 0.014 \\
\hline CD@ 5\% & 0.039 & 0.030 & 0.041 & 0.035 & 0.038 & 0.059 & 0.027 & 0.038 & 0.043 & 0.044 \\
\hline \multicolumn{11}{|c|}{ Two factor interaction } \\
\hline $\mathbf{V}_{1} \mathbf{T}_{1}$ & 7.64 & 8.03 & 7.43 & 7.62 & 7.47 & 7.74 & 7.90 & 8.63 & 7.66 & 7.98 \\
\hline $\mathbf{V}_{1} \mathbf{T}_{2}$ & 7.03 & 7.42 & 7.06 & 7.24 & 7.34 & 7.53 & 7.14 & 7.64 & 7.13 & 7.47 \\
\hline $\mathbf{V}_{2} \mathbf{T}_{1}$ & 7.72 & 8.02 & 8.02 & 7.94 & 7.84 & 8.23 & 7.52 & 7.92 & 7.78 & 8.02 \\
\hline $\mathbf{V}_{2} \mathbf{T}_{2}$ & 7.44 & 7.73 & 7.72 & 7.70 & 7.60 & 7.70 & 7.42 & 7.73 & 7.56 & 7.65 \\
\hline $\operatorname{SEm}( \pm)$ & 0.018 & 0.013 & 0.019 & 0.016 & 0.017 & 0.027 & 0.012 & 0.017 & 0.020 & 0.020 \\
\hline CD@ 5\% & 0.055 & 0.042 & NS & 0.049 & 0.054 & 0.083 & 0.039 & 0.054 & 0.062 & 0.062 \\
\hline
\end{tabular}

$\mathrm{A}=$ Ambient $\left(25 \pm 2{ }^{0} \mathrm{C}\right), \mathrm{R}=$ Refrigerated $\left(5 \pm 2{ }^{0} \mathrm{C}\right)$

$\mathrm{V}_{1}$ : Sardar guava variety (white flesh), $\mathrm{V}_{2}$ : Lalith guava variety (Pink flesh).

$\mathrm{T}_{1}: 750 \mathrm{~g}$ sugar $+5 \mathrm{~g}$ salt $+2 \mathrm{~g}$ citric acid per kg guava pulp, $\mathrm{T}_{2}: 750 \mathrm{~g}$ sugar per $\mathrm{kg}$ guava pulp 
Plate.1 Sardar guava leather at 0 days storage

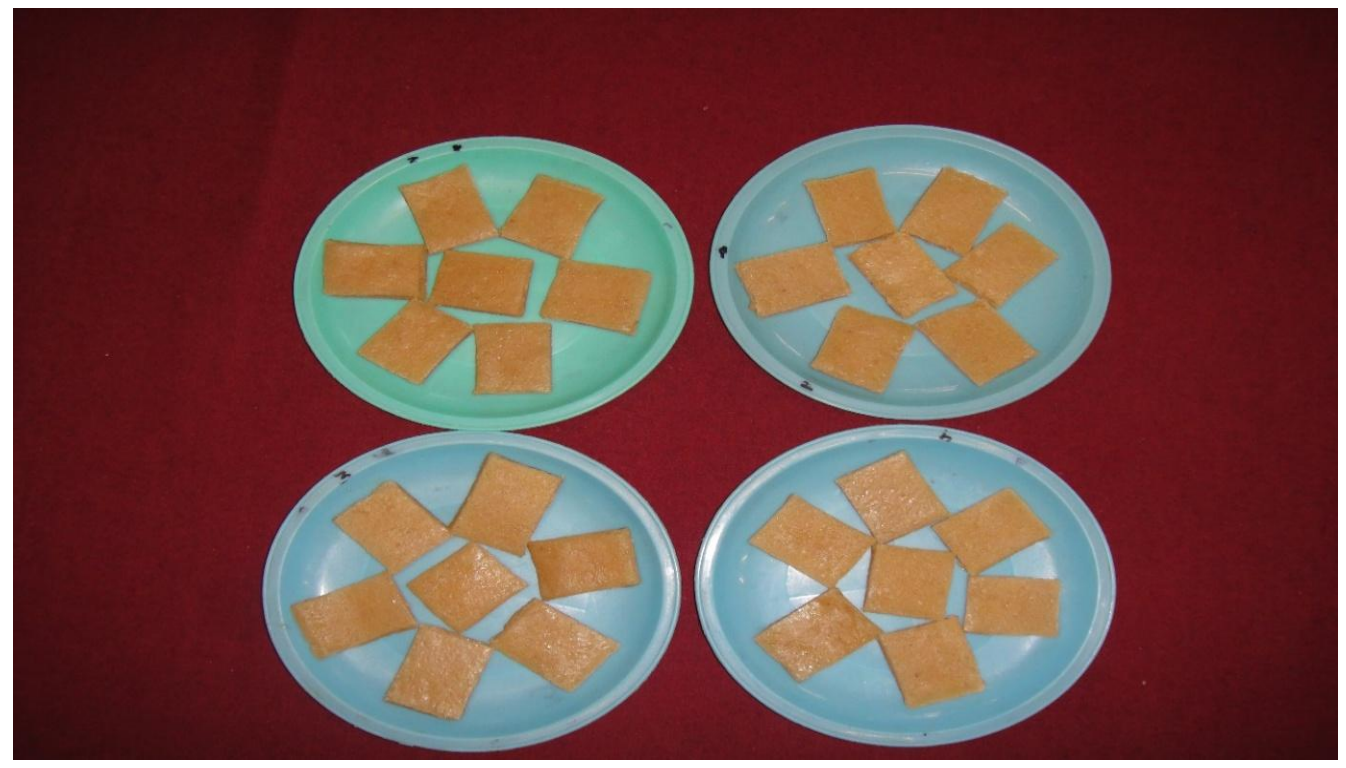

Plate.2 Lalith guava leather at 0 days storage

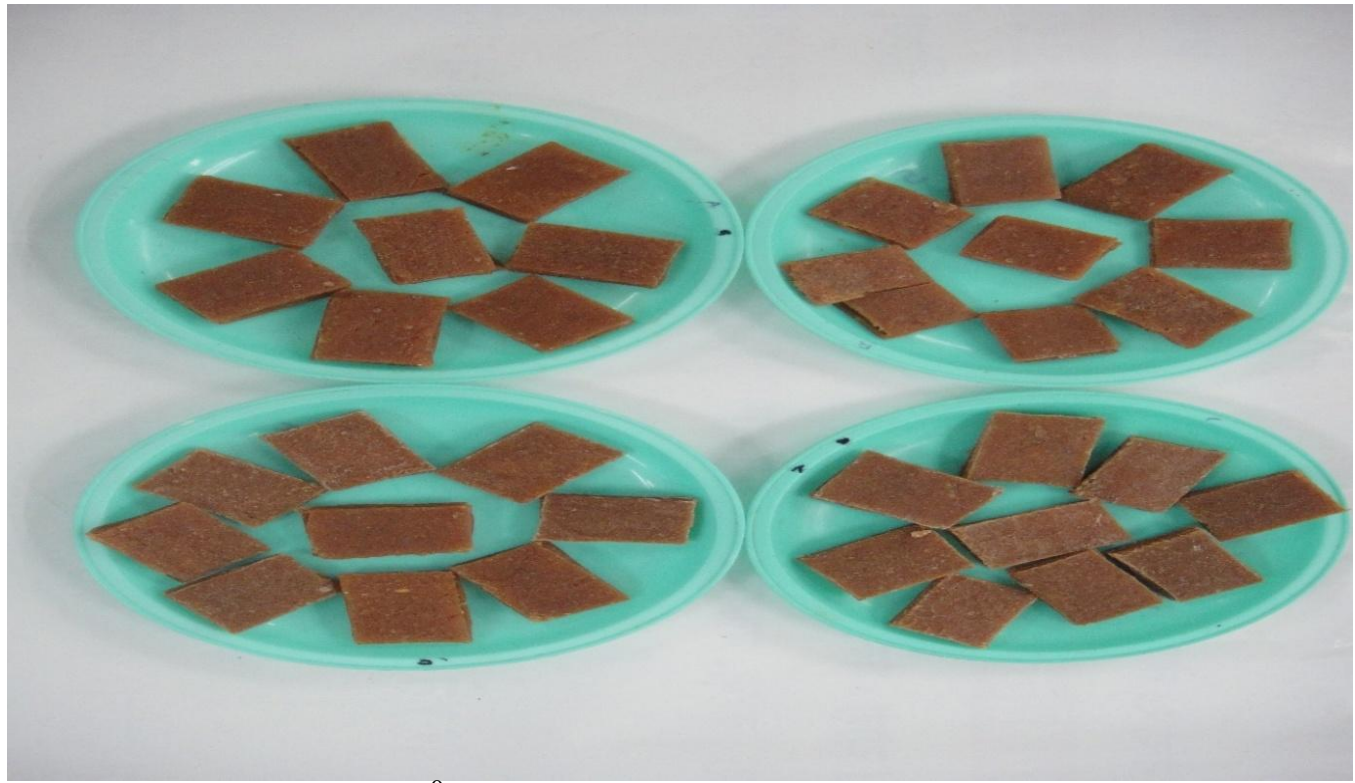

1-2, Ambient temperature $\left(27 \pm 2{ }^{0} \mathrm{C}\right)$

$3-4$, Refrigerated temperature $\left(7 \pm 2{ }^{0} \mathrm{C}\right)$

The maximum score 8.27 was observed in $\mathrm{V}_{2} \mathrm{~T}_{1}$ sample stored at refrigerated temperature.

The score for texture decreased significantly during storage at ambient temperature than stored at refrigerated temperature. A gradual decrease in texture score is due to hardening effect resulting from loss of moisture during storage.

Similar results were obtained in various experiments performed earlier on sweet potato leather (Collins and Hutsell, 1987), 
jack fruit leather (Che Man and Taufik, 1995), fig and other fruit products (Doreyappa Gowda et al., 1995). Guava -papaya fruit bar (Vennilla et al., 2004), fig leather (Kotlawar, 2008) and high protein tamarind leather (Kharche, 2012). The results obtained in the present investigation for guava leathers are in agreement with literature.

\section{Taste}

The results on taste score of guava leather samples are presented in Tables 9-12. A gradual decrease in score for taste of guava leathers from 8.30 to 7.49 at ambient temperature and from 8.30 to 7.98 at refrigerated temperature was observed.

The taste deterioration was more in guava leathers at ambient condition than at refrigerated temperature. The guava leather samples of $\mathrm{V}_{2} \mathrm{~T}_{1}$ at refrigerated temperature gave maximum taste score 8.60 in comparison with other treatments. This might be due to proper blending of sugar and acidity as well as consistency of the guava leather. Both conditions of storage for guava leather samples gave acceptable taste score.

It is reported that the taste score decreased during storage with respect to storage condition and period of storage., in sweet potato leather (Collins and Hutsell, 1987), jack fruit leather (Che Man and Taufik, 1995), guava -papaya fruit bar (Vennilla et al., 2004) and fig leather (Kotlawar, 2008).

The results obtained in the present investigation for taste score in guava leathers are in agreement with literature.

\section{Overall acceptability}

The results on overall acceptability score of guava leathers influenced by storage condition and period with respect to various treatments are presented in Tables 9-12 A gradual decrease in overall acceptability score from 8.38 to 7.53 at ambient temperature and from 8.38 to 7.78 at refrigerated temperature. It was observed that decrease in overall acceptability score at ambient temperature was faster than at refrigerated temperature.

The maximum score of 8.00 was observed in treatment $\mathrm{V}_{2} \mathrm{~T}_{1}$ stored at refrigerated temperature.

The highest overall acceptability score was observed in guava leathers stored at refrigerated temperature than at ambient temperature. It may be due to faster deterioration in terms of color, flavor, texture and taste at higher temperature during ambient condition. It is reported that the overall acceptability score decrease during storage with respect to the storage condition and period.

Previous researchers have shown that the decrease in overall acceptability in case of papaya leather (Harvey and Cavaletto, 1978), mango fruit bar (Doreyappa Gowda et al., 1994), jack fruit leathers (Che Man and Taufik, 1995), guava-papaya fruit bar (Vennilla et al., 2004) and mango leather (Gill et al., 2004).

\section{Texture analysis of guava leathers}

The Texture analysis of guava leathers was done at initial 0 days storage and final after 90 days storage by using the available Shimazdu Texturometer. The force in $(\mathrm{N})$ used to break down the individual leather is recorded separately. The results obtained are presented in Table 8. Results obtained stated that more force was used to break the fresh leather and less force was used after 90 days storage. It may be all due to increase in crystallization of sugar within increase in storage period. 


\section{Economics for making guava leathers}

The results on production cost of guava leathers are presented in Table 6. The cost of production of $1 \mathrm{~kg}$ guava (white flesh) leather of treatment $\mathrm{T}_{1}$ was Rs.135.55 and for treatment $\mathrm{T}_{2}$ Rs. 135.00 only.

Whereas, the cost of production of $1 \mathrm{~kg}$ guava (pink flesh) leather was of treatment $\mathrm{T}_{1}$ was Rs.155.55 and for treatment $\mathrm{T}_{2}$ Rs. 155.00 only. These costs did not include rent, transport charges, sale commission, local taxes etc. However, there was no significant difference in cost of guava leathers making among the treatments. The costs are for laboratory (small scale) preparation of guava leathers. These may be still reduced during mechanization of the process for mass production.

From the results of this research it was concluded that in physicochemical analysis, guava leather prepared with treatment $\mathrm{T}_{1}$ $(750 \mathrm{~g}$ sugar $+5 \mathrm{~g}$ salt $+2 \mathrm{~g}$ citric acid per $\mathrm{kg}$ guava pulp) showed better organoleptic properties as well as good storage stability at both storage (ambient and refrigerated) conditions up to 3 months storage period Microbial results also stated that product was safe to consume even after 3 months at both storage temperatures

\section{References}

Amerine, M.A., Pangborn, R.M. and Osler, E.B. 1965. Principles of sensory evaluation of food. Academic press, New York. pp. 350-480.

A.O.A.C. 2005. Official Methods of Analysis. 18th Edn. Association of Official Analytical Chemists, Virginia, USA. pp. 321.

Balasubramanian, S. 2007. Studies on textural properties of selected pulps and its retexturised fruit bars. Beverage Food World. 34(2): 67-69.

Bhagirathi, B., Rao, V. and Shrihari, K. 1993. Microbiological studies on the production of dehydrated convenience foods. J. Food Sci. Technol. 30(6): 403-407.

Bijay Kumar, 2011. Indian Horticulture Database- 2010. National Horticulture Board 124-127

Chavan, U.D., Pawar, U.B. Pawar, G.H. March 2015. Studies on preparation of mixed toffee from guava and strawberry. J. Food Sci. Technol., DOI 10.1007/s13197-015-1786-3.

Chavan U.D., Kohinkar S.N.2012. Osmotic dehydration process for preservation of fruits and vegetables. 1(2): 202.

Che Man, Y.B. and Taufik.1995. Development and stability of jack fruit leather. Tropical; Sci. 35(3): 245-250.

Collins, J.L. and Hutsell, L.W. 1987. Physical, Chemical, Sensory and Microbiology attributes of sweet potato leather. J. Food Sci., 52.

DAC (Department of Agriculture and cooperation). 2007. Ministry of Agriculture, Govt. of

Doreyappa Gowda, I.N., Amba Dan and Ramanjaneya, K.H. 1995. Studies on mango fruit bar preparation. Indian Food Packer. March-April 17.

FAO (1983). Production yearbook, Rome.

Gawade, M.H. and Waskar, D.P. 2003. Studies on processing and storage of fig fruits. J. Maharashtra Agric. Univ. 28(2): 148-150.

Gill, B.S., Sodhi, N.S., Singh, C.N., Singh, M. and Singh, D. 2004. Effects of Brix, sodium alginate and drying temperature on color texture and sensory properties of 'Dashehari' mango leather, J. Food Sci. Technol. 41(4): 373-378. 
*Harrigan, W.F. and Mccance, M.E. 1966. Laboratory Methods in Microbiology. Academic press. London. pp. 14-16.

Harvey, T.C. Jr. and Cavaletto, G. 1978. Dehydration and storage stability of Papaya leather. J. Food Sci.43: 17231725.

Jagtiani, J., Chan, H.T. and Sakai, W.S. 1998. Guava in tropical fruit processing, Academic Press, New York. pp. 9-44

Jain, P.K. and Asati, V.K.2004. Evaluation of guava cultivars for pulp preparation. J.Food Sci.Technol. 41(6):684-686.

Joshi, A. 2009. Studies on processing and value addition of sweet tamarind (variety- Ajanta) M.Sc. (Agri.) Thesis. M.A.U Parbhani.

Kharche, K.M. 2012. Studies on preparation of high protein leather from tamarind. M.Sc.

Kohinkar S.N., Chavan U.D., Pawar U.D., and Amarowicz. R. 2014. Studies on preparation of mixed fruit toffee from fig and guava fruits. J. Food Sci. Technol. 51(9), 2204-2209.

Kotlawar, N.G. 2008. Investigations on preparation and shelf life of fig leather. M.Sc. Thesis., M.P.K.V., Rahuri.

Krishnaveni, A., Manimegalai, G., Venilla, P. and Saravanakumar, R. 1999. Storage stability of jackfruit bar in different packaging materials. Indian Food packer. 53(6): 67-71.

Lane, J.H. and Eynon, L. 1923. Determination of sugars by Fehling solution with methylene blue as internal indicator. $J$. Soc. Chem. India.42:32-34.

Leite, K.M.S.C., Tadiotti, A.C., Baldochi, D. and Oliveria, O.M.M.F. (2006). Partial purification, heat stability and kinetic characterization of the pectin methylesterase from Brazilian guava Plauma cultivars. Food Chemistry. 94, 565-572.
*Le Maguer, M. 1988. Osmotic dehydration, Review and future directions. In Proc. International symposium on progress in food preservation and processes. Brussels, Belgium, 1: 283-309.

Mir, M.A, and Nirankarnath, M. 1993. Storage changes in fortified mango bars. J.Food Sci. Technol. 30(4):279282.

Misra, K. and Sheshadri, T.R. 1968. Chemical components of fruits of guava. Phytochemistry. 7(4): 641.

Mitra, K., Ghosh, S.K. and Dhua, R.S. 1984. Ascorbic acid content in different varieties of guava grown in West Bengal. Sci. Cult. 50: 235.

Muhammad NaeemSafdar. 2014.Quality of guava leather as influenced by storage period and

Panse, V.G. and Sukhatme, P.V. 1967. Statistical Methods for Agril. Workers, Indian Council of Agril Research, New Delhi. pp. 70-72.

Pawar, S.G., Kulkarni, D.N., Shere, D.M., Kulkarni, K.d., Paril, V.K. 1992. Effect of pretreatments on chemical composition and drying rates of solar dried figs.

Ranganna, S. 1986. Handbook of Analysis and Quality Control for Fruit and vegetable Products. McGraw-Hill Tata Pub. Co. Ltd., New Delhi. pp. 7-12 and 109.

Rao, M.A. 1977. Energy consumption in refrigerated canned and frozen peas. $J$. Food Proc. Engg.1: 149-169.

Rao, S.V. and Roy S.K. 1980a. Studies on dehydration of mango pulp. I Standardization of making mango sheets/ leather. Indian Food Packer.34 (3): 64-71.

Rao, S.V. and Roy S.K. 1980b. Studies on dehydration of mango pulp. II Storage studies of mango sheets/leather. Indian Food Packer. 34 (3): 72-79. 
Rathore, D.S. 1976. Effects of season on growth and chemical composition of guava fruits. J. Hort. Sci. 51 (1): 41.

RozinaNaz., 2012. Physical properties, sensory attributes and consumer preference of fruit

Singh, M. 1988. Performance of some cultivars of guava with special reference to their commercial significance in the central gangetic plains. Punjab Hort. J. 28 (1 and 2): 50.

Somogyi, L. P., Barret, D.M. and Hui, Y.H. 1996. Major processed product. 2 US: Technomic Publishing Co. Inc.
Thonta, G.T. and Patil, V.K. 1988. Studies on drying of fig fruits Indian Food Packer.42 (4): 94-99.

Vennilla, P. 2004. Studies on storage behavior of guava-papaya fruit bar. Beverage Food world. 31(2): 63-66.

Verma, A.R. and Shrivastava, J.C. 1965. Pectin in guava during growth and maturity. Indian J. Hort. 22 (1 and 2): 318.

Yadav, I. S. 1997. Search for quality cultivars, The Hindu, Survey of Indian Agriculture. Pp. 132.

Yadav. P.K. 2007. Handbook of Fruit Production Technology, pp. 223-240.

\section{How to cite this article:}

Shaik Jakeer Basha, Er., A. Yerri Swamy, Lingala Ramu and Sreenivas, D. 2018. Organoleptic Properties of Guava Fruit Leather with Effectiveness of Increase in Storage Period. Int.J.Curr.Microbiol.App.Sci. 7(05): 2226-2242. doi: https://doi.org/10.20546/ijcmas.2018.705.260 